\title{
Bronchial Artery Embolization for Hemoptysis
}

\author{
Manrita Sidhu, M.D., 1,2,4 Karen Wieseler, M.D., ${ }^{2}$ Thomas R. Burdick, M.D., ${ }^{2,3}$ \\ and Dennis W. W. Shaw, M.D. ${ }^{1,2}$
}

Bronchial artery bleeding is the most common cause of life-threatening hemoptysis. The most common underlying etiologies include tuberculosis, bronchiectasis, aspergillosis, and cystic fibrosis. Bronchial artery embolization is an important treatment for significant hemoptysis, given its high early success rate and relatively low risk compared with alternative medical and surgical treatments. In this article, the relevant anatomy and pathophysiology leading to bronchial artery bleeding is discussed, including the roles of parenchymal lung diseases and of collateral and aberrant vessels. The indications for treatment, success rate, and complication rate for bronchial artery embolization are reviewed. Preprocedure clinical stabilization and evaluation, including the roles of radiographs, bronchoscopy, and computed tomography examination are evaluated. Details of technique, including the published variety of approaches, and an emphasis on avoidance of nontarget embolization of important mediastinal structures and of the anterior spinal artery are discussed.

KEYWORDS: Hemoptysis, bronchial artery, embolization, selective angiography

Objectives: On completion of this article, the reader should be familiar with the anatomical and clinical considerations and the safe performance of bronchial artery embolization for the treatment of life-threatening hemoptysis.

Accreditation: Tufts University School of Medicine (TUSM) is accredited by the Accreditation Council for Continuing Medical Education to provide continuing medical education for physicians.

Credit: TUSM designates this educational activity for a maximum of 1 AMA PRA Category 1 Credit ${ }^{\mathrm{TM}}$. Physicians should only claim credit commensurate with the extent of their participation in the activity.

\section{CLINICAL PRESENTATION}

Although hemoptysis is a relatively common presenting symptom in patients seen by pulmonary specialists, lifethreatening massive hemoptysis is rare, accounting for $\sim 5 \%$ of patients hospitalized with hemoptysis. ${ }^{1}$ The definition of hemoptysis is expectorated blood arising from the pulmonary parenchyma or tracheobronchial tree. ${ }^{1}$ Ninety percent of significant hemoptysis is of bronchial artery origin. ${ }^{2}$ Because the alternative to medical treatment of massive hemoptysis carries a mortality rate of 50 to $100 \%$ and surgical treatment a mortality rate of up to $40 \%$ in the emergent setting, bronchial artery embolization (BAE) has become a well-established palliative or curative therapy for significant hemoptysis. ${ }^{1-5}$

\section{Definition and Indications for Treatment}

A variety of definitions have been proposed for the term "massive hemoptysis," the most commonly used being the expectoration of greater than $240 \mathrm{~mL}$ ( $\sim 1$ cup) in
${ }^{1}$ Children's Hospital and Regional Medical Center; ${ }^{2}$ University of Washington School of Medicine; ${ }^{3}$ Harborview Medical Center, Seattle, Washington; ${ }^{4}$ Seattle Radiologists, Advanced Imaging Center, Everett, Washington.

Address for correspondence and reprint requests: Manrita Sidhu, M.D., Seattle Radiologists, Advanced Imaging Center, 3927 Rucker
Avenue, Everett, WA 98201 (e-mail: manritasidhu@gmail.com). Embolization 2008; Guest Editor, Thomas R. Burdick, M.D.

Semin Intervent Radiol 2008;25:310-318. Copyright (C) 2008 by Thieme Medical Publishers, Inc., 333 Seventh Avenue, New York, NY 10001, USA. Tel: +1 (212) 584-4662.

DOI 10.1055/s-0028-1085931. ISSN 0739-9529. 
24 hours. ${ }^{6}$ However, the reliability of patient measurement and reporting of volume of expectorated blood may be limited in real life, and a reasonable practical definition of massive is any amount of hemoptysis that is life-threatening. ${ }^{7-9}$ The morbidity and mortality of this disease is due to asphyxiation in $80 \%$ of cases, and exsanguination in the remainder. ${ }^{10}$ In addition to massive hemoptysis, lesser degrees of hemoptysis may also require treatment, depending on the patient's underlying pulmonary reserve and ability to maintain a patent airway. ${ }^{2}$ Ongoing chronic but nonmassive hemoptysis that impairs a patient's quality of life or performance of essential physical therapy or pulmonary toilette may also require endovascular treatment. ${ }^{11,12}$ Lastly, episodes of milder hemoptysis may be a precursor of a lifethreatening massive hemoptysis event. ${ }^{1}$ Therefore, some authors have advocated elective treatment of severely respiratorily compromised patients - defined as a forced expiratory volume at 1 second $(\mathrm{FEV}-1)<35 \%$ predicted-following mild episodes of hemoptysis because this may be predictive of a potentially fatal future episode of major hemoptysis. ${ }^{6}$ In addition to hemoptysis, other indications for BAE include pre- or postoperative embolization, treatment of congenital lesions, and posttraumatic bleeding. ${ }^{10,13}$ Although BAE has a relatively high short-term success rate, if the etiology of the hemoptysis is an uncontrolled or uncontrollable progressive disease, bronchial artery embolization may be considered a palliative, rather than curative, procedure. ${ }^{5}$

\section{Risk Factors and Etiologies}

The most common etiology for hemoptysis in the developing world is tuberculosis. ${ }^{4}$ In the developed world, the most common etiologies are primary lung neoplasm, bronchiectasis, aspergillosis, and cystic fibrosis. $^{2}$ In children, after cystic fibrosis, congenital cardiac or vascular abnormalities are other causes. ${ }^{13}$ Other risk factors or etiologies associated with the occurrence of massive hemoptysis include coagulopathic and immunocompromised states, congenital or acquired heart disease, chronic pulmonary emboli, aneurysms, fistulas, interstitial fibrosis, history of smoking, pulmonary endometriosis, trauma (including iatrogenic), and chronic inflammatory conditions. ${ }^{1,2,7,14}$

\section{Other Etiologies for Hemoptysis}

Although not discussed in this article, aortobronchial fistula is an uncommon etiology for massive hemoptysis, with stent-graft placement and surgery as treatment options. ${ }^{12}$ Bronchial artery aneurysm has been reported, presenting as a mediastinal mass and resected at thoracotomy. ${ }^{15}$ Pulmonary artery arteriovenous malformations are another important cause of hemoptysis. Evaluation and treatment are highly specialized, mainly due to the association with hereditary hemorrhagic telangiectasia and risk of paradoxic embolus. The reader is referred to publications detailing this topic. ${ }^{10,14,16-18}$

\section{THE DISEASE}

\section{Pathophysiology}

The lungs have a dual vascular supply system including the pulmonary and the bronchial arteries, with multiple anastomoses providing an extensive collateral network between the two systems. ${ }^{1,19}$ The two systems communicate extensively via capillary beds near the alveoli and produce a right-to-left shunt that normally accounts for $5 \%$ of cardiac output. ${ }^{1,20,21}$ The bronchial circulation is responsible for the $90 \%$ of cases of massive hemoptysis. Approximately $5 \%$ of cases are due to abnormalities of the pulmonary circulation and another 5\% from systemic vessels or vessels directly arising from the aorta. ${ }^{2}$ The pulmonary arteries and veins are a relatively low-pressure system, arising from the right heart, which takes part in gas exchange and makes the greatest contribution to pulmonary blood supply. The bronchial arteries, from a high-pressure system, provide nourishment to mediastinal, hilar, and pulmonary structural tissues and account for $\sim 1 \%$ of pulmonary blood supply. Structures supplied include the vasa vasorum of the pulmonary circulation vessels, lymph nodes, and visceral pleura. ${ }^{1}$ Bronchial vessels branch extensively around the bronchi they supply and form a plexus in the peribronchial and submucosal bronchial space, ending in the terminal bronchioles. ${ }^{1}$ Bronchial vessels also have been shown to anastomose with branches of the subclavian, internal mammary, pericardiophrenic, esophageal, intercostal, and other systemic arteries. ${ }^{19}$ These connections are important in both understanding the pathophysiology of hemoptysis and the potential risks of embolotherapy.

Conditions that cause pressure derangements in these arterial systems can result in anatomical changes that predispose to hemoptysis. Several possibilities for the pathophysiology of hemoptysis exist. ${ }^{1,19,20,22,23}$ The underlying initiating factor is abnormal decreased pulmonary arterial perfusion to the lung, secondary to occlusion or vasoconstriction, either in response to local lung parenchymal abnormality due to intrinsic abnormality of the pulmonary vessel (such as with thromboembolic disease or vasculitis) or due to congenital cardiac conditions resulting in decreased pulmonary arterial supply (such as tetralogy of Fallot). ${ }^{24}$ The preexisting collateral circulation to the lungs provided by the bronchial arteries, under the higher systemic arterial pressure, can enlarge to compensate for the decreased pulmonary arterial perfusion. In addition, there may be neovascularity and vascular remodeling associated with inflammatory processes (bronchiectasis, chronic bronchitis, and chronic necrotizing infections) and neoplastic 
diseases. The bronchial arteries then form enlarged abnormal collateral vessels that, in the presence of inflammation or systemic arterial pressure, rupture into the airspaces, resulting in hemoptysis. ${ }^{1,19,22}$

The exception is in the case of increased left atrial pressure, such as in untreated mitral valve stenosis; the etiology is, rather, increased back pressure in the bronchial veins, which become variceal and can bleed, as in "cardiac apoplexy."

\section{ANATOMY}

\section{Bronchial Arteries (Orthotopic)}

The main function of the bronchial arteries is to supply bilateral bronchial trees. Bronchial arteries are typically multiple and bilateral and arise from the descending thoracic aorta at or below the level of carina. ${ }^{25}$ The anatomy is variable from patient to patient, but the standard or orthotopic origin is from the aorta between the levels of T5 and T6. In 60 to $80 \%$ of patients, there is a single right bronchial artery, with its origin from the right posterolateral thoracic aorta, and in $90 \%$ of cases arising from an intercostal artery, also called an intercostobronchial trunk (ICBT) $)^{2,24}$ (Fig. 1). Left-sided bronchial arteries, as well as additional right-sided branches, if present, usually arise from the anterolateral thoracic aorta. They are more frequently multiple and less likely to arise as a common ICBT. ${ }^{2,10}$ A common origin of bilateral bronchial arteries can be seen in $\sim 45 \%$ of patients ${ }^{10}$ (Fig. 2). Four branching patterns have been described using cadaver data ${ }^{26}$ : In $40 \%$, there were two left bronchials and an ICBT on the right; in 21\%, there were an ICBT on the right and a single left bronchial; in $20 \%$, there were two left bronchials, one bronchial on the right, and a right ICBT; in 9.7\%, there were one bronchial on the left, one bronchial on the right, and a right ICBT. Uflaker et al have reported an expanded angiographically derived classification, with 10 different branching patterns. ${ }^{27}$

\section{Anomalous Bronchial Arteries}

Bronchial arteries arising outside of T5-T6 are considered anomalous, with an incidence of 8.3 to $35 \%{ }^{11,28}$ These can arise from many vessels in the thorax, including thoracic or abdominal aorta, brachiocephalic and subclavian arteries or branches (Fig. 3), intercostal arteries, celiac artery branches, and phrenic arteries (Fig. 4). These are identified as bronchial vessels because they parallel the course of the bronchi once within the thorax.

\section{Systemic Arteries}

Systemic arteries do not follow the major bronchi but are acquired collateral vessels that enter the lung from

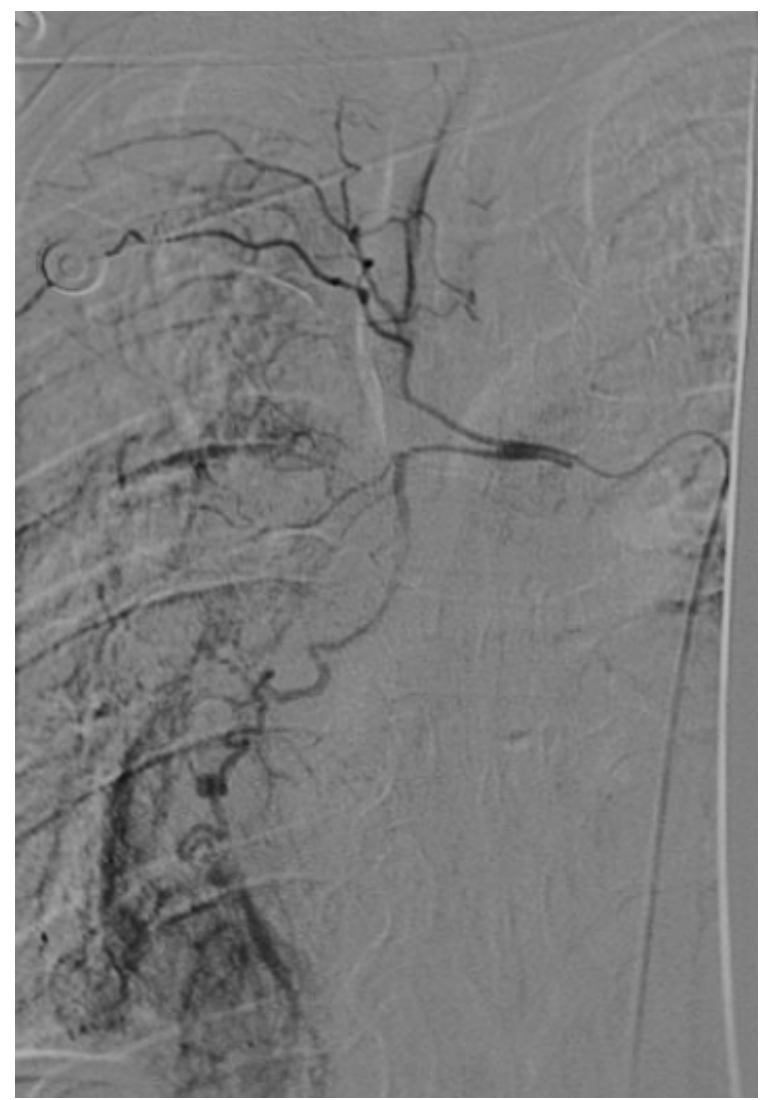

Figure 1 Right bronchial arteries arising from a right intercostobronchial trunk.

adjacent abnormal pleura or pulmonary ligament. ${ }^{2}$ Transpleural collaterals can arise from a variety of vessels including intercostals, subclavian, and internal mammary vessels. Subdiaphragmatic inferior phrenic, left gastric, and aortic branches can be relevant sources of collaterals. ${ }^{12}$ Pleural thickening or fibrosis is associated with an increased incidence of systemic collaterals. ${ }^{2,29,30}$

\section{Spinal Artery}

The most feared complication of bronchial artery embolization is spinal cord stroke. This risk exists because the anterior spinal artery or artery of Adamkiewicz can originate from a bronchial artery in up to $5 \%$ of patients, more often on the right side. ${ }^{23}$ On computed tomography $(\mathrm{CT})$ or conventional angiography, this is seen as a small longitudinal vessel on the mid ventral surface of the spinal cord. ${ }^{31}$ Immediately after its origin from the aorta it has a characteristic hairpin turn before reaching the ventral surface of the cord (Fig. 5).

\section{Other Systemic Collaterals}

Bronchial arteries have normally existing collaterals which have been experimentally shown to be $325 \mu \mathrm{m}$ 


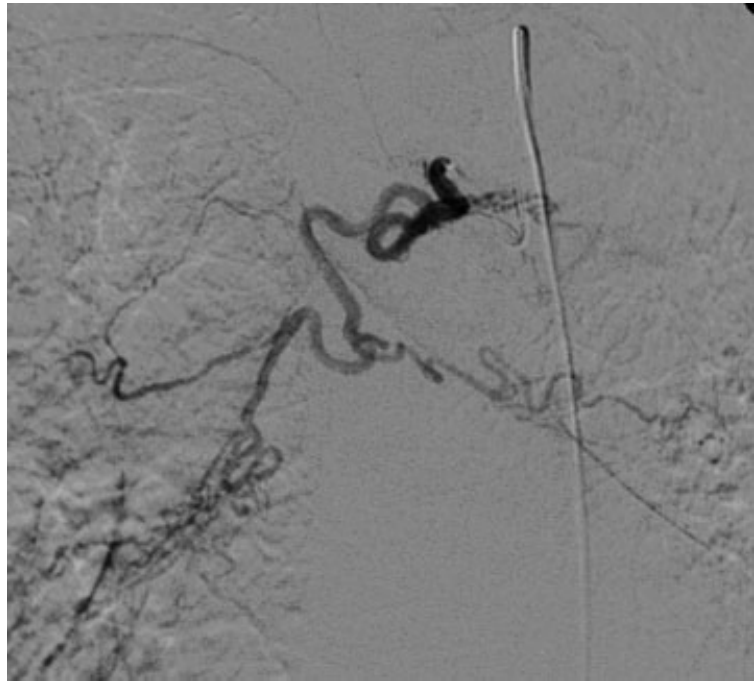

Figure 2 Bronchial angiogram showing a common origin of bilateral bronchial arteries.

in diameter to pulmonary arteries and pulmonary veins. ${ }^{21}$ Bronchial arteries also normally supply peripheral branches to the bronchi, esophagus, vasa vasorum of the aorta, and pulmonary arteries. ${ }^{2}$ Therefore, the use of embolic agents that are smaller than $350 \mu \mathrm{m}$ should be avoided to avoid damaging these small but important collaterals. Inadvertent embolization of these collaterals has the potential for systemic embolizations, esophageal infarction, or necrosis of the wall of the

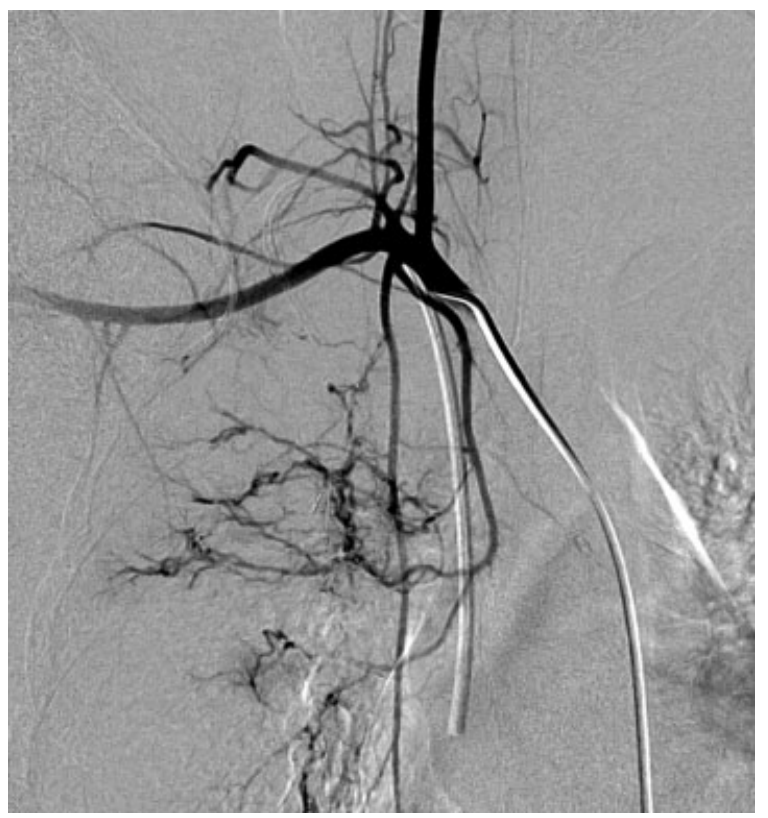

Figure 3 Right subclavian angiogram demonstrating an anomalous right bronchial artery arising from the right internal mammary. (Image courtesy of Dr. Manraj Heran)

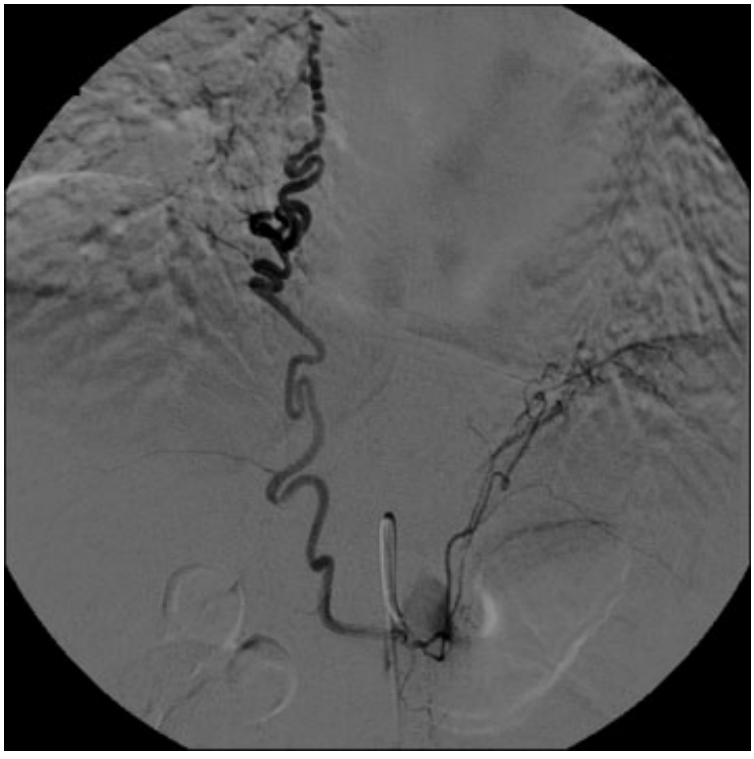

Figure 4 Phrenic angiogram demonstrating an anomalous right bronchial artery in this patient with recurrent hemoptysis.

aorta or pulmonary arteries, with potentially catastrophic consequences.

\section{EVALUATION}

\section{Contraindications}

The only real contraindications for bronchial artery embolization (BAE) are the usual contraindications, absolute or relative, for angiography in general including uncorrectable coagulopathy, renal failure, and severe contrast allergy. In congenital pulmonary artery stenosis, the bronchial collateral vessels may provide an essential role in pulmonary parenchymal perfusion, and therefore careful consideration and pediatric cardiology consultation is imperative in these cases before treatment.

\section{Clinical Considerations}

In the setting of life-threatening hemoptysis, it is imperative to medically stabilize the patient with measures including protecting the airway, optimizing oxygenation, supporting the patient hemodynamically, and correcting any coagulopathy that may be present. ${ }^{7}$ Due to risk of asphyxiation, protective airway maneuvers may be required before and during the procedure. These include keeping the bleeding side dependent to avoid aspiration of blood into the contralateral airway, selective intubation of the uninvolved lung if bleeding is lateralized, use of a dual lumen tube to separately ventilate the lungs, endobronchial tamponade with a Fogarty catheter, and topical hemostasis via bronchoscopy, if indicated. ${ }^{7,17}$ 


\section{Preprocedure Evaluation and Workup}

Preprocedure workup is performed with the goals of elucidating the cause of the hemoptysis and to guide location of endovascular therapy. ${ }^{2}$ Other sources of hemoptysis from the nasopharynx, gastrointestinal tract, or oropharynx should be excluded. ${ }^{1}$ Angiographic localization of the origin of the abnormal vessels can assist in a prompt, directed, and efficient procedure, with decreased time on the table and increased safety. Radiography, bronchoscopy, CT, and CT angiography have all been used. Conventional chest radiography is a quick, noninvasive, and widely available first step that can localize the site of abnormality in 19 to $83 \%$ of cases. ${ }^{2}$ Several authors report that a subjective sense of "a funny feeling" or "gurgling" in the chest, if reported by the patient, is highly predictive of the site of bleeding., ${ }^{6,10,11}$

Traditionally, bronchoscopy has been considered an important step before embolization, with the expectation that the site can be localized, leading to more rapid and directed embolization. In addition, there is the potential for treatment bronchoscopically when the etiology is an endobronchial lesion. Reported success of bronchoscopy in lateralizing the side of bleeding is 49 to $93 \% .^{2}$ However, in the setting of a normal radiograph, bronchoscopy only adds additional localizing information in 0 to $31 \%$ of cases. ${ }^{2}$ More recent reports suggest that bronchoscopy may be less useful than thought, due to low detection rate of location of bleeding in the setting of massive hemoptysis, little added benefit if location and etiology of bleeding is known radiographically, inherent risks of the procedure, and possible delay in definitive therapy. $6,11,32,33$

Computed tomography and CT angiography have increasingly been found useful in pre-embolization planning by locating the site of bleeding; underlying lung pathologies, if present; and abnormal vessels. ${ }^{25,30} \mathrm{Com}^{-}$ puted tomography can localize the site of bleeding in 63 to $100 \%$ of cases. $^{32,34}$ Specifically, CT can aid in diagnosing nonbronchial systemic supply in $80 \%$ of cases, with the lowest success in visualizing internal mammary supply. ${ }^{2}$ Knowledge of nonbronchial supply in advance has the potential for increasing the success rate of initial embolization. ${ }^{25,30} \mathrm{~A}$ variety of opinions exist regarding whether pre-embolization $\mathrm{CT}$ or bronchoscopy should be performed, with the final decision in each case requiring balancing the possibility of improved preprocedure information against possible delay in definitive treatment, especially in the setting of a lifethreatening condition. A practical approach may be CT angiography in a nonacute setting, with the goals of identifying the site and etiology of bleeding and determining the relevant anatomy, especially localization of the artery of Adamkiewicz. ${ }^{31}$ In the setting of hemodynamic instability or acute airway compromise, the best approach would likely be chest radiograph followed directly by angiography with embolization.

\section{ENDOVASCULAR THERAPY}

\section{Approach and Technique}

The first report of BAE to control hemoptysis was published by Rémy et al in $1974 .{ }^{35}$ In 1976 , this was followed by Wholey et al's report of four cases and then the first large series by Rémy et al in 1977. ${ }^{4,36}$ Since then, there have been numerous publications with a variety of suggested algorithms for workup and embolization of hemoptysis. A widely accepted and relatively conservative approach is to treat ipsilaterally enlarged bronchial arteries at the first session. In the event of recurrent hemoptysis following technically successful embolizations, a search can then be made for a nonbronchial systemic artery supply. ${ }^{8,25}$ This approach limits the risk of nontarget embolization but may increase the number of procedures necessary to achieve hemostasis. In the case of congenital hemoptysis, however, systemic origin should be sought initially. ${ }^{25}$ Others suggest a more comprehensive approach at the first treatment setting, including treatment of all visualized bronchial arteries, not just ipsilateral, or with a search for systemic arteries at the first setting, after treatment of involved bronchial arteries. ${ }^{6,12}$

Another point of discussion is whether to begin with thoracic aortography or whether to directly commence selective bronchial artery angiography. ${ }^{6,12}$ One published approach is to perform selective bronchial arteriography at initial treatment and, for recurrent hemoptysis after technically successful embolizations, to perform thoracic and subclavian arteriography. In the setting of lower lobe disease, inferior phrenic evaluation should also be performed. ${ }^{6}$ Clearly, if no abnormal bronchial arteries are found at initial treatment, then systemic evaluation should be performed. ${ }^{6}$

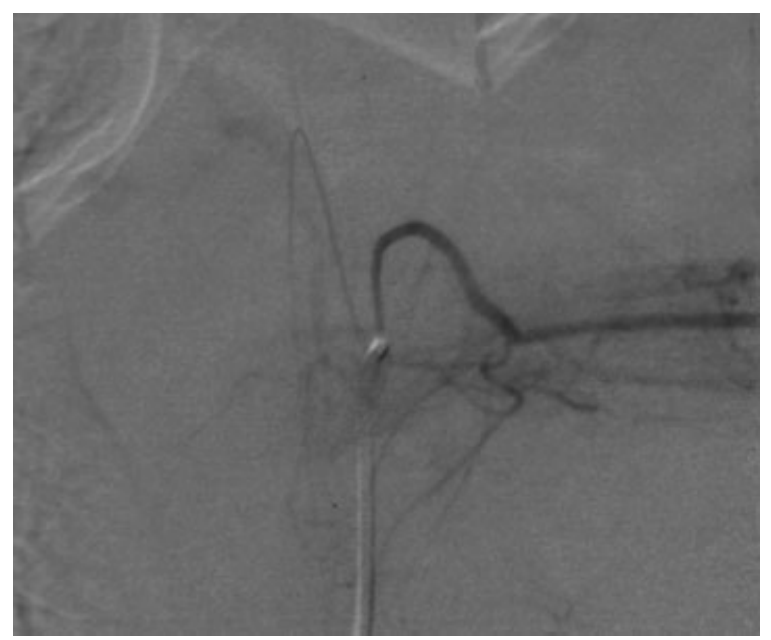

Figure 5 Typical appearance of an anterior spinal artery (artery of Adamkiewicz) arising from a left intercostal artery. 
Before embolization, high-quality diagnostic bronchial angiograms should be performed and carefully examined for presence of the anterior spinal artery to avoid inadvertent embolization. If findings are equivocal, an oblique view may be useful. ${ }^{11}$ If the anterior spinal artery does arise from an affected bronchial artery, careful distal embolization of that bronchial artery should only be performed if stable catheter position can be obtained distal to the origin of the spinal artery. ${ }^{23}$ Several authors have noted that spinal artery branches arising from bronchial arteries may not be visible until after embolization of the bronchial artery is completed, likely due to the small size of the spinal branches. This is a finding that confirms the need to embolize distally under fluoroscopic visualization; to use particles $>350 \mu \mathrm{m}$; and to avoid embolization past stasis, which would allow reflux of embolic material proximally. ${ }^{5,10}$

A variety of $4 \mathrm{~F}$ or $5 \mathrm{~F}$ catheters can be used for selection of the bronchial arteries. The tip is placed at the top of the descending thoracic aorta and directed right posterolaterally to search for the right ICBT and left anterolaterally to search for left bronchials. ${ }^{24,37}$ Because the right ICBT is the most likely of the bronchial vessels to supply the spinal artery, the catheter should not be wedged, as is generally true in all angiography. Hand injections of 5 to $10 \mathrm{cc}$ of contrast can be performed at each selected bronchial vessel. If abnormal findings are present, embolizations can be performed - with care being taken to embolize distally in the vessel-using a coaxial $3 \mathrm{~F}$-microcatheter technique, if necessary. The use of subselective embolizations via coaxial microcatheters may improve the success rate and lower the complication rate. ${ }^{38}$ Use of somatosensory-evoked potentials has also been proposed to increase safety. ${ }^{39}$

Following stable catheter placement, embolization proceeds under continuous fluoroscopic visualization until flow in the vessel is significantly slow. At that point, the remaining embolic material is flushed with saline with the goal of achieving distal stasis in the vessel while avoiding reflux backward into normal circulation. Proximal occlusion should be carefully avoided in bronchial artery embolotherapy. ${ }^{37}$ Next, the descending thoracic aorta is evaluated between T4 and T8. Embolization of all visualized abnormal bronchial arteries should be performed.

If necessary and depending on approach, at this point bilateral subclavian angiography may be performed, especially in the case of recurrence. A thoracic aortogram may be used if indicated. If lower lobe origin is suspected, a lower thoracic/upper abdominal aortogram may help determine the phrenic artery source. ${ }^{37}$ If no bronchial artery source is found, a pulmonary angiogram should be performed to exclude entities such as pulmonary artery aneurysms, arteriovenous malformations, and fistulas. ${ }^{24}$

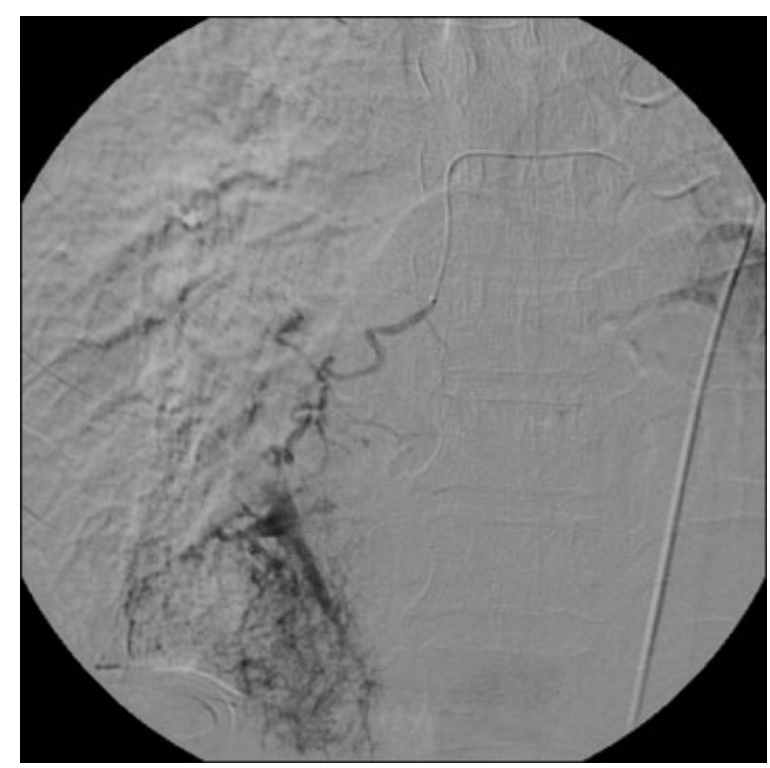

Figure 6 Right bronchial angiogram demonstrating significant shunting to the right lower lobe pulmonary artery branches.

\section{Angiographic Diagnosis}

Angiographic findings indicating embolization of the affected vessel include: hypervascularity (83\%), tortuous and enlarged bronchial arteries (31\%), shunting into pulmonary artery or vein (28\%) (Fig. 6), parenchymal staining (15\%), vascular abnormalities such as aneurysms (13\%), and extravasation into the airway (2 to $10.7 \%)^{23,25,32,40}$ (Fig. 7). Bronchial arteries larger than 2 to $2.5 \mathrm{~mm}$ in diameter are considered enlarged. ${ }^{2,11}$

\section{Embolic Agents}

The most widely used embolic agents are polyvinyl alcohol particles of 350 to $500 \mu \mathrm{m}$ and gelatin sponge pledgets. Polyvinyl alcohol particles are preferred to gelatin sponge pledgets because the pledgets are resorbable and may not provide as durable a result as the permanent agents. ${ }^{24}$ Microspheres have also been reported as successful embolizations material. ${ }^{41}$ To avoid nontarget damage to small normal collaterals and the risk of aortic, esophageal, bronchial, or pulmonary artery wall necrosis, smaller particles or liquid embolic agents should be avoided. ${ }^{2}$ Coils should be avoided to preserve access to future sites of bronchial bleeding and allow retreatment of an affected vessel. ${ }^{2}$ Proximal coil placement limits future embolizations to a series of what are usually progressively smaller and less accessible collateral vessels, until the arterial supply to the target tissue cannot be catherized. Coils may, however, have a role in the treatment of arterial aneurysms or pseudoaneurysms or in occlusion 


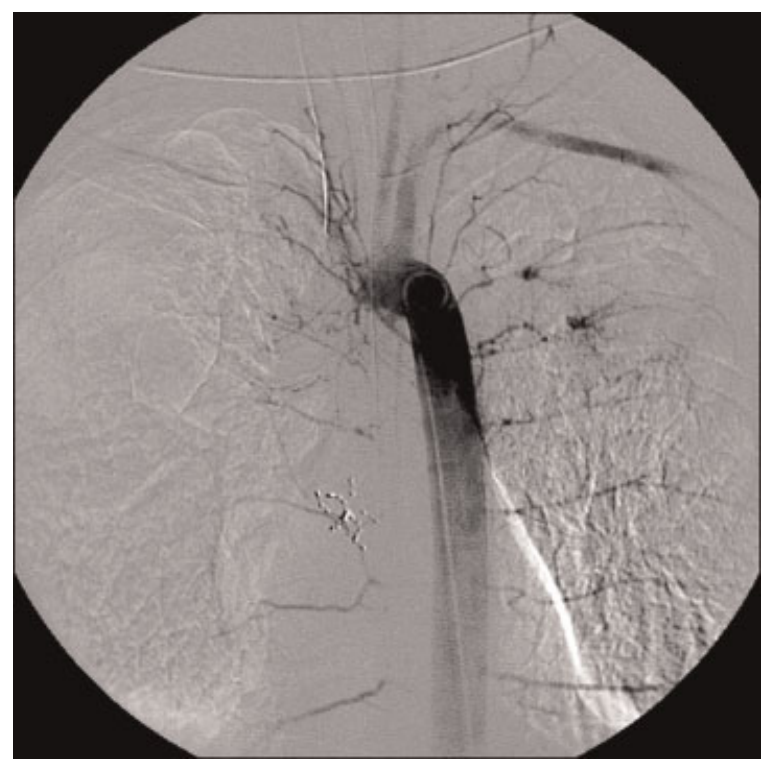

Figure 7 Descending thoracic aortogram illustrating the uncommon finding of extravasation from left bronchial arteries.

of an internal mammary artery to protect normal territory and avoid backfilling via collaterals. ${ }^{2,42,43}$

\section{Success Rate}

Technical success rates range from 65 to $100 \%{ }^{12,23}$ More recent reports have had higher technical success rates of 90 to $94 \%$, likely due to increased use of superselective techniques and increased use of permanent, rather than absorbable, embolization materials. ${ }^{10,41,44}$ Recurrent bleeding can be seen in 10 to $50 \%$ of patients. ${ }^{10,12}$ The short-term bleeding recurrence rate, within 1 month, is reported to be from 10 to $49 \%{ }^{12,23,41}$ Greater than 30 -day recurrence rates range between 10 and $52 \% .{ }^{10,42,45}$ Causes for recurrent bleeding include: progression of underlying disease; recanalization or revascularization; incomplete embolization; and emergence of other supply to the affected area, such as from systemic collaterals. ${ }^{2}$ Not surprisingly, recurrence rates depend on the underlying etiology of the lung disease, being higher in the settings of infections such as chronic tuberculosis (15.5\%), with aspergilloma (75\%), and in neoplasms (42\%) and better in bronchiectasis. ${ }^{2,10,46,47}$ In one series of 18 patients with cystic fibrosis, hemoptysis was controlled with the first embolization in $75 \%$, after the second session in $89 \%$, and after the third in $93 \%$ of patients. In this series, the chances of the source of bleeding arising from nonbronchial systemic collaterals was $75 \%$ in patients who had recurrent bleeding versus $8 \%$ in those being treated for the first time. ${ }^{6}$ It is likely that this represents successful embolization of the bronchial artery supply, with later recruitment of systemic collaterals in a chronic disease.

\section{Complications}

The true complication rate is difficult to asses due to heterogeneity of underlying disease and technique. However, more recent series report few complications, which is likely due to improvement in contrast materials and technique. ${ }^{23,24}$ Rare cases of paraplegia, transient paraplegia, and transient Brown-Séquard syndrome have been reported following bronchial artery embolizations, with an overall risk of transverse myelitis of less than $1 \%{ }^{8,12,38,40}$ Postembolization syndrome, consisting of retrosternal chest pain, intercostal pain, transient dysphagia, and fever is not uncommon. ${ }^{24}$ Other reported complications include stroke, esophageal ulceration, bronchial infarction and stenosis, bronchoesophageal fistula, transient left orbital and forehead pain, and transient neurological symptoms. . $^{60,12,40,41}$ Because spinal cord branches are often not visualized until after embolizations of the bleeding, suggesting that there are often small, nonvisualized spinal cord branches, subselective microcatheter techniques may have lower risk of complications. $^{5,38,46}$

\section{CONCLUSIONS}

Acute massive hemoptysis or chronic recurrent hemoptysis is rare but potentially life-threatening conditions, with the majority caused by hypertrophied bronchial arteries in response to a pulmonary parenchymal abnormality. Bronchial artery embolization is a well-established treatment with an excellent short-term success rate and an acceptable long-term success rate in what are often chronic diseases with limited surgical options. With appropriate technique it is a safe and well-tolerated procedure with a better outcome than medical, surgical, or bronchoscopic techniques alone.

\section{REFERENCES}

1. Thompson AB, Teschler H, Rennard SI. Pathogenesis, evaluation, and therapy for massive hemoptysis. Clin Chest Med 1992;13(1):69-82

2. Yoon W, Kim J, Kim Y, Chung T, Kang H. Bronchial and nonbronchial systemic artery embolization for life-threatening hemoptysis: a comprehensive review. Radiographics 2002;22(6):1395-1409

3. Magilligan DJ, Ravipati S, Zayat $P$, et al. Massive hemoptysis: control by transcatheter bronchial artery embolization. Ann Thorac Surg 1981;32(4):392-400

4. Rémy J, Arnaud A, Fardou H, Giraud R, Voisin C. Treatment of hemoptysis by embolization of bronchial arteries. Radiology 1977;122(1):33-37 
5. White RI. Bronchial artery embolotherapy for control of acute hemoptysis: analysis of outcome. Chest 1999;115(4): 912-915

6. Brinson GM, Noone PG, Mauro MA, et al. Bronchial artery embolization for the treatment of hemoptysis in patients with cystic fibrosis. Am J Respir Crit Care Med 1998;157(6 Pt 1): 1951-1958

7. Håkanson E, Konstantinov I, Fransson S, Svedjeholm R. Management of life-threatening haemoptysis. Br J Anaesth 2002;88(2):291-295

8. Mal H, Rullon I, Mellot F, et al. Immediate and long-term results of bronchial artery embolization for life-threatening hemoptysis. Chest 1999;115(4):996-1001

9. Stoller J. Diagnosis and management of massive hemoptysis: a review. Respir Care 1992;37(6):564-581

10. Najarian KE, Morris CS. Arterial embolization in the chest. J Thorac Imaging 1998;13(2):93-104

11. Cohen AM, Doershuk CF, Stern RC. Bronchial artery embolization to control hemoptysis in cystic fibrosis. Radiology 1990;175(2):401-405

12. Andersen PE. Imaging and interventional radiological treatment of hemoptysis. Acta Radiol 2006;47(8):780792

13. Fellows KE, Khaw KT, Schuster S, Shwachman H. Bronchial artery embolization in cystic fibrosis; technique and long-term results. J Pediatr 1979;95(6):959963

14. Jean-Baptiste E. Clinical assessment and management of massive hemoptysis. Crit Care Med 2000;28(5):16421647

15. Tringali S, Tiffet O, Berger J, Cuilleret J. Bronchial artery aneurysm disguised as a leiomyoma of the esophagus. Ann Thorac Surg 2002;73(2):632-633

16. Pelage JP, El Hajjam M, Lagrange C, et al. Pulmonary artery interventions: an overview. Radiographics 2005;25(6):16531667

17. Saluja S, Henderson K, White RJ. Embolotherapy in the bronchial and pulmonary circulations. Radiol Clin North Am 2000;38(2):425-448 ix

18. Pollak JS, Saluja S, Thabet A, et al. Clinical and anatomic outcomes after embolotherapy of pulmonary arteriovenous malformations. J Vasc Interv Radiol 2006;17(1):35-44; quiz 45

19. Liebow AA, Hales MR, Lindskog GE. Enlargement of the bronchial arteries, and their anastomoses with the pulmonary arteries in bronchiectasis. Am J Pathol 1949;25(2):211231

20. Bruzzi JF, Rémy-Jardin M, Delhaye D, et al. Multi-detector row CT of hemoptysis. Radiographics 2006;26(1):322

21. Pump KK. Distribution of bronchial arteries in the human lung. Chest 1972;62(4):447-451

22. Ellis K. Fleischner lecture. Developmental abnormalities in the systemic blood supply to the lungs. AJR Am J Roentgenol 1991;156(4):669-679

23. Swanson KL, Johnson CM, Prakash UB, et al. Bronchial artery embolization: experience with 54 patients. Chest 2002; 121(3):789-795

24. Roberts AC. Bronchial artery embolization therapy. J Thorac Imaging 1990;5(4):60-72

25. Rémy-Jardin M, Bouaziz N, Dumont P, et al. Bronchial and nonbronchial systemic arteries at multi-detector row CT angiography: comparison with conventional angiography. Radiology 2004;233(3):741-749

26. Cauldwell E, Siekert R, Lininger R. The bronchial arteries: an anatomic study of 150 cadavers. Surg Gynecol Obstet 1948; 86:395-412

27. Uflacker R, Kaemmerer A, Picon P, et al. Bronchial artery embolization in the management of hemoptysis: technical aspects and long-term results. Radiology 1985;157(3):637644

28. Sancho C, Escalante E, Domínguez J, et al. Embolization of bronchial arteries of anomalous origin. Cardiovasc Intervent Radiol 1998;21(4):300-304

29. Tamura S, Kodama T, Otsuka N, et al. Embolotherapy for persistent hemoptysis: the significance of pleural thickening. Cardiovasc Intervent Radiol 1993;16(2):85-88

30. Yoon W, Kim Y, Kim J, et al. Massive hemoptysis: prediction of nonbronchial systemic arterial supply with chest CT. Radiology 2003;227(1):232-238

31. Takase K, Sawamura Y, Igarashi K, et al. Demonstration of the artery of Adamkiewicz at multi- detector row helical CT. Radiology 2002;223(1):39-45

32. Hsiao EI, Kirsch CM, Kagawa FT, et al. Utility of fiberoptic bronchoscopy before bronchial artery embolization for massive hemoptysis. AJR Am J Roentgenol 2001;177(4): 861-867

33. Sweezey NB, Fellows KE. Bronchial artery embolization for severe hemoptysis in cystic fibrosis. Chest 1990;97(6):13221326

34. Abal AT, Nair PC, Cherian J. Haemoptysis: aetiology, evaluation and outcome-a prospective study in a third-world country. Respir Med 2001;95(7):548-552

35. Rémy J, Voisin C, Dupuis C, et al. Traitement des hemoptysies par embolisation de la circulation systemique. Ann Radiol (Paris) 1974;17:5-16

36. Wholey MH, Chamorro HA, Rao G, Ford WB, Miller WH. Bronchial artery embolization for massive hemoptysis. JAMA 1976;236(22):2501-2504

37. Stoll JF, Bettmann MA. Bronchial artery embolization to control hemoptysis: a review. Cardiovasc Intervent Radiol 1988;11(5):263-269

38. Tanaka N, Yamakado K, Murashima S, et al. Superselective bronchial artery embolization for hemoptysis with a coaxial microcatheter system. J Vasc Interv Radiol 1997;8(1 Pt 1): 65-70

39. Schrodt JF, Becker GJ, Scott JA, Warren CH, Benenati SV. Bronchial artery embolization: monitoring with somatosensory evoked potentials. Work in progress. Radiology 1987;164(1): 135-139

40. Ramakantan R, Bandekar V, Gandhi M, Aulakh B, Deshmukh H. Massive hemoptysis due to pulmonary tuberculosis: control with bronchial artery embolization. Radiology 1996;200(3):691-694

41. Corr PD. Bronchial artery embolization for life-threatening hemoptysis using tris-acryl microspheres: shortterm result. Cardiovasc Intervent Radiol 2005;28(4):439441

42. Marshall TJ, Jackson JE. Vascular intervention in the thorax: bronchial artery embolization for haemoptysis. Eur Radiol 1997;7(8):1221-1227

43. Karmy-Jones R, Hastreiter D, Burdick T. Hemothorax complicating bronchial artery aneurysm. Can Respir J 2005; 12(5):279-281 
44. Bredin CP, Richardson PR, King TK, et al. Treatment of massive hemoptysis by combined occlusion of pulmonary and bronchial arteries. Am Rev Respir Dis 1978;117(5):969973

45. Kato A, Kudo S, Matsumoto K, et al. Bronchial artery embolization for hemoptysis due to benign diseases: immediate and long-term results. Cardiovasc Intervent Radiol 2000;23(5):351-357
46. Yu-Tang Goh P, Lin M, Teo N, En Shen Wong D. Embolization for hemoptysis: a six -year review. Cardiovasc Intervent Radiol 2002;25(1):17-25

47. Hayakawa K, Tanaka F, Torizuka T, et al. Bronchial artery embolization for hemoptysis: immediate and long-term results. Cardiovasc Intervent Radiol 1992;15(3):154-158; discussion 158-159 